\title{
Intrathecal Administration of Nusinersen Using the Ommaya Reservoir in an Adult with 5q-Related Spinal Muscular Atrophy Type 1 and Severe Spinal Deformity
}

\author{
Vasileios Papaliagkas ${ }^{a} \quad$ Nikolaos Foroglou $^{\mathrm{b}}$ Petros Toulios $^{\mathrm{c}}$ \\ Maria Moschou ${ }^{d}$ Maria Gavriilaki ${ }^{d}$ Konstantinos Notas ${ }^{e}$ \\ Evangelia Chatzikyriakou ${ }^{e}$ Georgia Zafeiridoue $^{\mathrm{e}}$ Marianthi Arnaoutoglou $^{\mathrm{e}}$ \\ Vasilios K. Kimiskidis ${ }^{d}$ \\ aDepartment of Biomedical Sciences, School of Health Sciences, International Hellenic \\ University, Thessaloniki, Greece; ${ }^{b}$ 1st Department of Neurosurgery, AHEPA University \\ Hospital, School of Medicine, Aristotle University of Thessaloniki, Thessaloniki, Greece; \\ c424 General Military Hospital, Thessaloniki, Greece; d1st Department of Neurology, AHEPA \\ University Hospital, School of Medicine, Aristotle University of Thessaloniki, Thessaloniki, \\ Greece; 'Laboratory of Clinical Neurophysiology, AHEPA University Hospital, School of \\ Medicine, Aristotle University of Thessaloniki, Thessaloniki, Greece
}

Keywords

Adult spinal muscular atrophy · Nusinersen - Ommaya reservoir · Drug administration routes . Case report

\begin{abstract}
Spinal muscular atrophy (SMA) is a hereditary neuromuscular disorder, typically caused by survival motor neuron 1 (SMN1) gene deletion in chromosome $5 q$ resulting in loss of SMN protein. SMA type 1 progresses rapidly leading to increased mortality usually before the age of 2 years. Nusinersen, the first approved disease-modifying treatment for all 5q-SMA types and ages, is an antisense oligonucleotide administered intrathecally via repeated lumbar punctures. However, adult SMA patients typically present with severe scoliosis and spinal deformity. We present a 28-year-old patient with SMA type 1 and severe spinal deformity, who received nusinersen via a subcutaneously implanted Ommaya reservoir connected with an intrathecal catheter at the thoracic level. The repetitive administrations were completed uneventfully, obviating the need for repeated laborious lumbar punctures and eliminating
\end{abstract}


radiation exposure. In adult SMA patients, performing recurrent lumbar punctures can be technically challenging raising the need for an alternative route of administration. The use of Ommaya reservoirs is a viable, practical for repeated infusions, and safe option for the intrathecal delivery of nusinersen for select cases such as an adult SMA type 1 survivor with severe spinal deformity.

(C) 2021 The Author(s).

Published by S. Karger AG, Basel

\section{Introduction}

Spinal muscular atrophy (SMA) is an autosomal recessive genetic disorder, characterized by progressive degeneration of motor neurons in the anterior horns of the spinal cord and brainstem nuclei typically due to a deletion in exon 7 of chromosome $5 q 13.2$ [1, 2]. Disease severity varies according to the homologous SMN2 gene copy number with increasing copy numbers leading to a milder phenotype. SMA type 1 or Werdnig-Hoffmann disease has the most devastating clinical course. Patients with SMA type 1 present symptoms before 6 months of age, never sit independently, and have a 32\% survival rate at 2 years [3].

Until recently, SMA management was based exclusively on providing physical therapy, supportive nutritional and respiratory care, and treating emerging complications of muscle weakness and spinal deformity [4]. The first effective disease-modifying treatment, nusinersen, received FDA (Food and Drug Administration) and EMA (European Medicines Agency) approval 3 years ago and drastically changed SMA standards of care [5]. Nusinersen is an antisense oligonucleotide administered intrathecally in 5q-SMA patients of all ages and types requiring repeated dosing. Individuals with SMA, particularly adults, often present spinal deformities or scoliosis which raise technical difficulties in the intrathecal delivery of nusinersen using recurrent lumbar punctures, which is currently regarded as the gold standard technique. In order to facilitate intrathecal access, fluoroscopy, ultrasound, or CT guidance has been advocated and successfully employed [6,7]. On the other hand, in the long-term management of this life-long disease, the risk of excessive radiation exposure needs to be considered. The Ommaya reservoir might provide an alternative approach in select cases by securing constant access to the intrathecal space while avoiding the risk of excess radiation exposure and obviating the need for repetitive and technically demanding lumbar punctures.

We describe the case of a 28-year-old man, a long-term survivor of genetically confirmed 5q-SMA type 1 with severe spinal deformity, who underwent intrathecal administration of nusinersen repeatedly using an Ommaya reservoir connected to a thoracic subdural catheter. The Ethics Committee of the University General Hospital of Thessaloniki AHEPA (Greece) approved the study protocol, and written informed consent was obtained from the patient and his family.

\section{Case Presentation}

\section{Clinical Picture}

A 28-year-old man (height: $120 \mathrm{~cm}$; body weight: $30 \mathrm{~kg}$ ) with a genetically confirmed 5q-SMA type 1 and 3 copies of the SMN2 gene was referred to our center for intrathecal nusinersen therapy. Onset of symptoms, including hypotonia and symmetric flaccid quadriparesis, was reported 5 months after birth. The patient never acquired the ability to sit independently. He was hospitalized numerous times for pneumonia, requiring escalation of respiratory

\section{Karger's}


Fig. 1. X-ray of the lumbar and thoracic spine depicts severe thoracic kyphosis and lumbar lordosis and elimination of the lumbar intervertebral spaces.

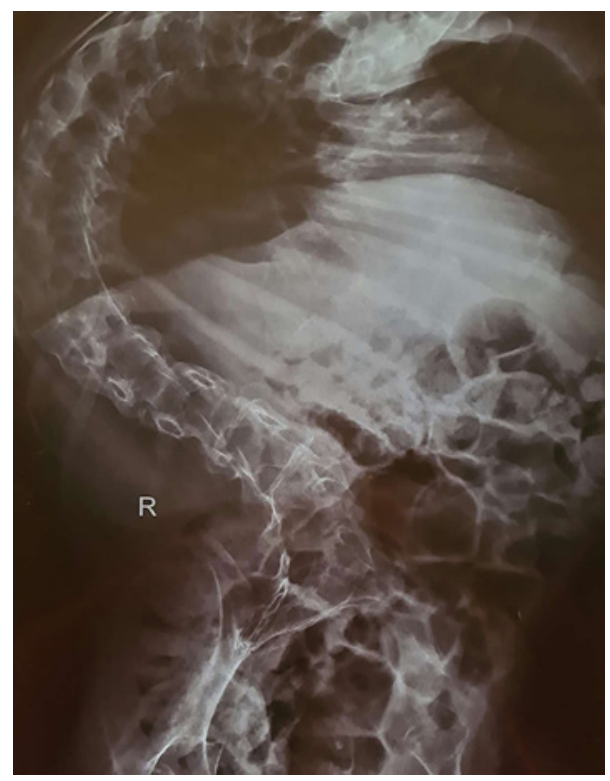

support with continuous bilevel positive airway pressure ventilation. Tracheostomy was performed at the age of 16 , following hospitalization in an intensive care unit.

His physical examination showed severe generalized hypotonia, significant generalized muscle atrophy and weakness, joint contractures, and areflexia. The patient was immobilized, unable to sit or even move any limb. Initial Revised Upper Limb Module (RULM) and Children's Hospital of Philadelphia Infant Test of Neuromuscular Disorders (CHOP-INTEND) scores were zero. He is using a PC equipped with speech recognition software.

His pulmonary function tests at first assessment revealed severe respiratory muscle weakness and severe restrictive lung disease (FEV1 15\% and FVC 18\%) with the patient being totally dependent on ventilatory support. An X-ray examination revealed severe kyphosis and lumbar lordosis as well as elimination of the lumbar intervertebral spaces (Fig. 1).

\section{Ommaya Reservoir Placement}

In view of the overall condition and, particularly, the severe spinal deformity of the patient, a multidisciplinary team concluded that the best individualized approach for the intrathecal administration of nusinersen would involve the implantation of a subcutaneous Ommaya reservoir stabilized at the lateral abdominal wall, $2 \mathrm{~cm}$ below the thoracic cage. The reservoir is connected to a catheter introduced intrathecally at the thoracic level through a minimal laminotomy. The catheter is fixed on the paraspinal structures in terms to avoid displacement. The operation was performed under a short-lasting general anesthesia in order to achieve adequate perioperative pain control and patient comfort, on a decubitus position.

The patient was discharged on the first postoperative day without any peri- or postprocedural complication. It should be noted that our patient was already dependent on a ventilator preoperatively, and therefore the risk of respiratory impairment as an effect of general anesthesia was not significant. The first dose of nusinersen was administrated uneventfully a week later. As of September 1, 2020, the patient had successfully received 6 doses of nusinersen through the Ommaya reservoir and remains in a stable condition with no untoward side effects.

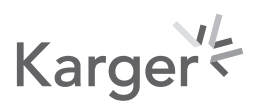


Papaliagkas et al.: Nusinersen Administration in an SMA Type 1 Adult

\section{Discussion}

The present case report describes a 28-year-old SMA type 1 patient with severe spinal deformity who was repeatedly administered nusinersen intrathecally via an Ommaya reservoir connected to a thoracic subdural catheter. The first notable feature of the reported case is the particularly prolonged disease course, which should be ascribed to the multidisciplinary, proactive management he received [8]. SMA type 1 patients are often dependent on respiratory and/or nutritional support from the age of 2. One of the first large studies that investigated Werdnig-Hoffmann disease's prognosis concluded that survival probability at 20 years of age was $0 \%$ [3]. In recent years, aggressive supporting care with mechanical ventilation use and gastrostomy tube feeding appears to be associated with prolonged survival [8]. However, this prolongation of life expectancy is not accompanied by improved neuromuscular functioning as natural history studies demonstrate a continuous decline in motor function scales, muscle strength, and maximal compound muscle action potential amplitudes of treatment-naïve patients with type 1 SMA, irrespective of SMN2 copy number [9].

Nusinersen, an antisense oligonucleotide, increases the production of normal SMN by modifying the splicing of the SMN2 gene. FDA and EMA approvals were mainly based on a sham-controlled clinical trial of nusinersen in SMA infants who were not older than 7 months of age $[5,10]$. Evidence of safety and efficacy in adults is based on real-world data from observational cohort studies [11]. Previous reports of nusinersen administration in adult 5q-SMA 1 survivors are scarce. However, it should be noted that in severely affected individuals with long disease duration, as in our patient, the evaluation of nusinersen's impact on functional outcome measures remains challenging. The 6-minute walk test is not clinically meaningful, and descriptive scales like the Hammersmith Functional Motor Scale Expanded or RULM could not detect marginal improvements in motor function of slowly progressive SMA disease in adults [12]. Hence, future studies should focus on finding more sensitive clinical biomarkers to measure drug efficacy in SMA patients of all types and ages.

One of the main challenges of nusinersen administration is the need for intrathecal access usually through recurrent lumbar punctures. SMA patients with long-standing disease often suffer from severe spinal deformities rendering this approach occasionally problematic. Indeed, since the approval of nusinersen, multiple real-world studies examined alternative methodologies including CT or fluoroscopy-guided lumbar puncture [13]. Other available approaches comprise cervical injections (with or without ultrasound or fluoroscopic guidance) and recently introduced subcutaneous intrathecal catheters or hybrid infusion systems $[14,15]$. It should be noted that in the context of CT and fluoroscopyguided approaches, there is a nonnegligible concern regarding exposure to excess radiation, particularly in view of the anticipated duration of treatment and the patients' young age [16]. On the other hand, Ommaya reservoirs are regarded as a secure and safe route of drug delivery mainly for chemotherapy. A literature review revealed 2 recent reports of Ommaya reservoir use as a means to nusinersen intrathecal administration in SMA patients. Lakhotia et al. [17] presented the successful, uncomplicated, off-label use of the Ommaya reservoir in a patient with SMA type 2 and concomitant scoliosis with spinal fusion. A recent retrospective cohort on intrathecal methods of nusinersen delivery in 1 institution reported that $22 \%$ of injections in patients with complicated spines were performed through Ommaya reservoirs placed in the lumbar region [18]. This cohort included both adults and children with SMA types 1-3.

Our case confirms and extends the findings of these 2 reports in an adult SMA type 1 case and demonstrates that the use of Ommaya reservoirs is a viable, practical for repeated infusions, and safe option for the intrathecal delivery of nusinersen in select cases. Further investigation of this approach in large-scale studies is warranted.

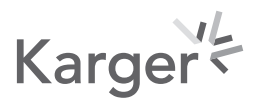


Case Reports in

Neurology

\begin{tabular}{l|l}
\hline Case Rep Neurol 2021:13:710-715 \\
\hline DOI: 10.1159/000519831 & $\begin{array}{l}\text { @ 2021 The Author(s). Published by S. Karger AG, Basel } \\
\text { www.karger.com/crn }\end{array}$ \\
\hline
\end{tabular}

Papaliagkas et al.: Nusinersen Administration in an SMA Type 1 Adult

\section{Acknowledgments}

We wish to thank the patient and his family for consenting to the publication of this case report.

\section{Statement of Ethics}

All procedures of this study followed the hospital's ethics committee standards and the Declaration of Helsinki. The study was approved by the Scientific Council of AHEPA University General Hospital of Thessaloniki (Greece) on January 31, 2019, Protocol No. 4080/2019. A written informed consent was obtained from the patient and his family for publication of this case report and any accompanying images.

\section{Conflict of Interest Statement}

The authors have no conflicts of interest to declare.

\section{Funding Sources}

No external funding was received for this study.

\section{Author Contributions}

All authors were involved in the patient's clinical care. V.K. and V.P. remain responsible for his ongoing monitoring. M.G. wrote the first draft manuscript. All other authors were involved in the revision process.

\section{Data Availability Statement}

All data generated or analyzed during this study are included in this article. Further enquiries can be directed to the corresponding author.

\section{References}

1 Cusco I, Barcelo MJ, Rojas-Garcia R, Illa I, Gámez J, Cervera C, et al. SMN2 copy number predicts acute or chronic spinal muscular atrophy but does not account for intrafamilial variability in siblings. J Neurol. 2006;253(1): 21-5.

2 Lefebvre S, Bürglen L, Reboullet S, Clermont O, Burlet P, Viollet L, et al. Identification and characterization of a spinal muscular atrophy-determining gene. Cell. 1995;80(1):155-65.

3 Zerres K, Rudnik-Schöneborn S. Natural history in proximal spinal muscular atrophy. Clinical analysis of 445 patients and suggestions for a modification of existing classifications. Arch Neurol. 1995;52(5):518-23.

4 Wang CH, Finkel RS, Bertini ES, Schroth M, Simonds A, Wong B, et al. Consensus statement for standard of care in spinal muscular atrophy. J Child Neurol. 2007;22(8):1027-49.

5 Finkel RS, Mercuri E, Darras BT, Connolly AM, Kuntz NL, Kirschner J, et al. Nusinersen versus sham control in infantile-onset spinal muscular atrophy. N Engl J Med. 2017;377(18):1723.

6 Ortiz CB, Kukreja KU, Lotze TE, Chau A. Ultrasound-guided cervical puncture for nusinersen administration in adolescents. Pediatr Radiol. 2019;49(1):136-40. 
7 Velayudhan V, Patel S, Danziger A, Grigorian A, Waite S, Efendizade A. Transforaminal lumbar puncture for intrathecal access: case series with literature review and comparison to other techniques. J Clin Neurosci. 2020;72:114-8.

8 Oskoui M, Levy G, Garland CJ, Gray JM, O'Hagen J, De Vivo DC, et al. The changing natural history of spinal muscular atrophy type 1. Neurology. 2007;69(20):1931-6.

9 Wijngaarde CA, Stam M, Otto LAM, Bartels B, Asselman FL, van Eijk RPA, et al. Muscle strength and motor function in adolescents and adults with spinal muscular atrophy. Neurology. 2020;95(14):e1988.

10 European Medicines Agency. First medicine for spinal muscular atrophy. Secondary first medicine for spinal muscular atrophy. 2017. Available from: https://www.ema.europa.eu/en/news/first-medicine-spinalmuscular-atrophy.

11 Hagenacker T, Wurster CD, Günther R, Schreiber-Katz O, Osmanovic A, Petri S, et al. Nusinersen in adults with 5q spinal muscular atrophy: a non-interventional, multicentre, observational cohort study. Lancet Neurol. 2020;19(4):317-25.

12 Main M, Kairon H, Mercuri E, Muntoni F. The Hammersmith functional motor scale for children with spinal muscular atrophy: a scale to test ability and monitor progress in children with limited ambulation. Eur J Paediatr Neurol. 2003;7(4):155-9.

13 Wurster CD, Winter B, Wollinsky K, Ludolph AC, Uzelac Z, Witzel S, et al. Intrathecal administration of nusinersen in adolescent and adult SMA type 2 and 3 patients. J Neurol. 2019;266(1):183-94.

14 Veerapandiyan A, Pal R, D'Ambrosio S, Young I, Eichinger K, Collins E, et al. Cervical puncture to deliver nusinersen in patients with spinal muscular atrophy. Neurology. 2018;91(7):e620-4.

15 Strauss KA, Carson VJ, Brigatti KW, Young M, Robinson DL, Hendrickson C, et al. Preliminary safety and tolerability of a novel subcutaneous intrathecal catheter system for repeated outpatient dosing of nusinersen to children and adults with spinal muscular atrophy. J Pediatr Orthop. 2018;38(10):e610-7.

16 Spiliopoulos S, Reppas L, Zompola C, Palaiodimou L, Papadopoulou M, Filippiadis D, et al. Computed-tomography-guided transforaminal intrathecal nusinersen injection in adults with spinal muscular atrophy type 2 and severe spinal deformity. Feasibility, safety and radiation exposure considerations. Eur J Neurol. 2020; 27(7):1343-9.

17 Lakhotia A, Bhalla S, Doll E, Gump W. Use of Ommaya reservoir with a thoracic spinal catheter for intrathecal delivery of nusinersen in a patient with spinal muscular atrophy type 2 (P4.464). Neurology. 2018;90(15 Supplement):P4464.

18 Cartwright MS, Ward ZT, White EP, West TG. Intrathecal delivery of nusinersen in individuals with complicated spines. Muscle Nerve. 2020;62(1):114-8. 